\title{
Characterization of TiO2-Chitosan/Glass photocatalyst for the removal of a monoazo dye via photodegradation-adsorption process
}

\begin{abstract}
In this paper, the newly explored $\mathrm{TiO} 2-$ Chitosan/Glass was suggested as a promising alternative material to conventional means of wastewater treatment. Characterization of TiO2-Chitosan/Glass photocatalyst was studied with SEM-EDX, XRD, and Fourier transform infrared spectroscopy (FTIR) analysis. The combination effect of photodegradation-adsorption process for the removal of methyl orange (MO), an acid dye of the monoazo series occur promisingly when four layers of TiO2-Chitosan/Glass photocatalyst was used for MO removal. Approximately, $87.0 \%$ of total MO removal was achieved. The reactive $-\mathrm{NH} 2,-\mathrm{OH}$, and metal oxide contents in the prepared photocatalyst responsible for the photodegradation-adsorption effect were confirmed by FTIR study. Similarly, MO removal behavior was well supported by SEM-EDX and XRD analysis. Significant dependence of MO removal on the TiO2-Chitosan loading can be explained in terms of relationship between quantum yield of photocatalytic reactions and photocatalyst structure/activity. Hence, the research work done thus far suggests a new method, having both the advantages of photodegradation-adsorption process in the abatement of various wastewater pollutants.
\end{abstract}

Keyword: Adsorption; Titanium dioxide; Chitosan; Visible light 